\title{
New Developments: Gastroenterology
}

\author{
Craig Hillemeier, MD
}

\section{Therapy of Inflammatory Bowel Disease}

Crohn's disease and ulcerative colitis cause significant morbidity in the pediatric age group. The precise etiology of inflammatory bowel disease remains unknown; therefore, our therapeutic efforts continue to be directed toward controlling the symptoms. Current therapy for inflammatory bowel disease is focused on the use of steroids because of their anti-inflammatory effect. Unfortunately, chronic steroid use is associated with significant and serious side effects such as increased blood pressure, fluid retention, bone thinning, and delayed growth. In an attempt to avoid these undesirable consequences, there has been a search for other antiinflammatory agents.

\section{6-Mercaptopurine and Azathioprine}

6-Mercaptopurine (6MP) and its precursor azathioprine have been shown to have benefits in treating inflammatory bowel disease. These agents are purine antagonists that are capable of inhibiting DNA and RNA synthesis and seem to be effective, especially when given for several months. They are cytotoxic to stimulated lymphoid cell lines, especially natural killer and suppressor cytotoxic cells. These effects are expressed clinically in adults with inflammatory bowel disease by a marked reduction in lymphocyte-mediated cytotoxicity, which is first seen after about 1 month of treatment and reaches a maximum effect after 4 to 5 months. This time interval corresponds closely to the period of time often required before beneficial effects of these medications are seen in patients with intractable Crohn's disease. It is important to note that these medicines may reduce steroid use over a long period of time but are not helpful in an acute exacerbation.

Although the steroid-sparing effects of these medications have been widely accepted in the treatment of adult patients with inflammatory bowel disease, clinicians responsible for care of pediatric patients with inflammatory bowel disease have been slow to adopt them. Fears of toxicity come from experience in treatment of childhood leukemias and suppression of rejection in transplant patients. However, in these conditions, such side effects as reversible bone marrow suppression, hepatotoxicity, and immune suppression are most commonly seen when 6MP is prescribed at doses of $>2 \mathrm{mg} / \mathrm{kg}$ per day, which is somewhat higher than most physicians use in treating pediatric patients with inflammatory bowel disease. Because these drugs interfere with DNA synthesis and exert a long-standing cytostatic effect on lymphoid cells, concerns have been raised about long-term risk of malignancies. However, studies in adult patients have not confirmed the risks. Experience with 6MP in the range that is used to treat inflammatory bowel disease, in the range of 1 to $2 \mathrm{mg} / \mathrm{kg}$ per day, is not widely available in the pediatric patient; however, preliminary studies in pediatric patients suggest that this drug is effective in reducing steroid dosage. ${ }^{1}$

$6 \mathrm{MP}$ needs to be studied in a controlled fashion to determine its efficacy and

Craig Hillemcicr, MD, is Director of Pediatric Gastroenterology at C. S. Mott Children's Hospital, University of Michigan Medical Center, Ann Arbor.

96/1/44866 
safety in the treatment of childhood inflammatory bowel disease, and these trials are currently under way. It is likely the steroid-sparing effects of these agents will be a welcome addition to the pharmacologic means of controlling childhood inflammatory bowel disease.

\section{Sulfasalazine and Its Derivatives}

Sulfasalazine is the acid azo compound of 5-aminosalicylic acid (5-ASA) and sulfapyridine. Sulfasalazine has been shown to be an effective agent in preventing and treating recurrence of ulcerative colitis and may have a therapeutic role in managing Crohn's disease. Its efficacy is restricted in $10 \%$ to $15 \%$ of pediatric patients because of the skin reaction to the sulfa component, and many patients experience significant gastrointestinal upset. Studies have shown that the salicylate portion of sulfasalazine is the therapeutically active component. This salicylate component likely has its therapeutic effect by interfering with prostaglandin and leukotriene synthesis, which are thought to be essential intermediates in the inflammatory process.

Because many of the adverse effects of Azulfidine are ascribed to the sulfa function of the compound, which is thought to be inactive, there have been recent attempts to delete the sulfa component. A variety of delivery vehicles for the salicylate moiety have recently been developed, and formulations of 5-ASA can be administered either orally or rectally. The salicylate preparation by itself has recently become available in an oral preparation of a 5 -aminosalicylate molecule bound to another 5-aminosalicylate molecule by a diazo bond without the sulfa component. Colonic bacteria then cleave this diazo bond in the colon, similar to Azulfidine, and the 5-ASA is liberated to act locally on inflamed colonic epithelium. Studies suggest similar efficacy to Azulfidine. This drug, known as dipentum, appears to be well tolerated, does not cause the immediate hypersensitivity skin reaction seen in response to the sulfa, appears to cause less gastrointestinal upset, and has been shown to be comparable in efficacy to Azulfidine in treating inflammatory bowel disease. ${ }^{2}$

Rectally administered formulations that allow a higher concentration of 5-ASA to be obtained in the rectum and recto-sigmoid than can be obtained with oral medication have been developed. They are currently available in the United States as a $4 \mathrm{gm}$ enema and $250 \mathrm{mg}$ suppositories. In adult patients, the rectal forms appear to be a valuable form of therapy in distal and left-sided colitis. Determination of the exact role and dosage of these 5-ASA preparations, both rectal and oral, in the management of children with inflammatory bowel disease is currently under way.

\section{Ulcer Disease Associated With Nonsteroidal Anti-Inflammatory Drugs}

Gastrointestinal tract ulceration associated with nonsteroidal anti-inflammatory drugs (NSAIDs) is commonly seen in pediatric populations in which such agents are chronically required. It has been estimated that the risk of a gastric ulcer in a patient on NSAIDs (i.e., aspirin) is about 40 times greater than that of the general population, and chronic ingestion of NSAIDs is associated with the development of mucosal ulceration in $10 \%$ to $20 \%$ of patients. Although these ulcers may occur at any time, they usually appear after at least 3 months of NSAID ingestion. It is hypothesized that these ulcers result from the systemic effects of the nonsteroidal anti-inflammatory drugs on the mucosal defense mechanisms. Some evidence suggests they result in decreased mucosal prostaglandin production. It is important to note that although many patients who develop mucosal inflam- 
mation on NSAIDs will have obvious abdominal symptoms, it is not infrequent for mucosal lesions to develop in the absence of overt gastrointestinal symptoms.

Although the ulcerations respond rapidly to conventional therapy if the agents are discontinued, in many patients this is not possible. H2-receptor antagonists may be successful in treating these lesions, but a prostaglandin E1 analogue, misoprostol, has been licensed in this country for the treatment of such lesions. The mechanisms by which these prostaglandins enhance mucosal protection include decreased gastric acid secretion, increased mucus secretion, bicarbonate secretion, blood flow, and epithelial regeneration. Although experience in children is limited, these prostaglandins have been demonstrated to be efficacious in the prevention of NSAID-associated gastric lesions in adults. ${ }^{3}$ The most common side effect of misoprostol is diarrhea and gastrointestinal upset.

Misoprostol also has been found to increase the efficiency of fat absorption in patients with cystic fibrosis. ${ }^{4}$ Although the precise mechanism is unclear, it is thought that the increased bicarbonate production seen with the prostaglandin analogue reduces intraluminal $\mathrm{pH}$ and allows the exogenously taken pancreatic enzyme to be more effective.

\section{Abdominal Pain, Ulcer Disease, and Helicobacter}

Although abdominal pain is a common complaint in pediatrics and peptic ulcer disease is frequently considered in the differential diagnosis, it is not common to find abdominal pain that is caused by clearly defined ulcers. When seen, gastric or duodenal ulcer disease is classified as either primary (idiopathic) or, more commonly seen, secondary to medications or systemic illness.

In evaluating patients with primary peptic ulcer disease, it has been found that many patients with duodenal ulcers, gastritis, or both will have evidence of bacterial colonization in the upper gastrointestinal tract. The strain was initially identified by its histologic appearance of silver stains of gastric antral biopsies. The organism, which was initially named a Campylobacter because of its morphologic resemblance to other Campylobacter bacteria, has been reclassified on the basis of genetic information as Helicobacter pylori.

Although most patients with primary duodenal ulcer disease have evidence of the organism H. pylori in the gastric antrum, it is not true that all patients with $H$. pylori in the gastric antrum will have ulcer disease. When this organism is present in the gastric antrum in the absence of duodenal ulcer disease, its association with pathology such as abdominal pain is less certain, but it has been associated with histologic evidence of gastritis. ${ }^{5}$ There is some evidence that in adults, the severity of symptoms correlates with the degree of histologic inflammation. One feature that has made it difficult to determine the precise relationship between the presence of this organism, gastritis, and clinical symptoms is that the histologic finding of gastritis by the pathologist on microscopic examination of antral biopies correlates very poorly with the presence or absence of inflammation of gastric mucosa as either seen by an endoscopist or evaluated by contrast radiography during an upper gastrointestinal series. To further complicate the issue, the presence of $H$. pylori and its relationship to abdominal pain symptoms must be interpreted with caution, because it has also been found in antral biopsies of patients with no gastrointestinal symptoms. It also is worthy to note that $H$. pylori in gastric antral tissue is seen increasingly with advancing age, and it is quite common to see it within the sixth decade of life, even in people with no gastrointestinal tract inflammation.

Currently a diagnosis of $H$. pylori is best made by histologic examination of the gastric biopsy, although a gel test that turns color in response to the increased 
urease activity of the organism is helpful, and increasingly sensitive serologic tests for antibodies to Helicobacter are becoming available. The treatment of possible $H$. pylori must be undertaken with caution, because the relationship between clinical symptoms and the actual presence of the organism is oftentimes unclear, especially in the absence of duodenal ulcer disease. Triple therapy, which consists of a bismuth compound, antibiotics, and $\mathrm{H} 2$ antagonist, has been shown to eradicate the organism in a high percentage of cases.

\section{Hepatitis}

During the past 2 years, it has become apparent that a large number of hepatitis cases thought to be infectious in etiology but without supporting serologic evidence for hepatitis $A$ or $B$ are caused by a virus now known as hepatitis $C$. Hepatitis $C$ virus contains a positive-strand RNA molecule and appears to be related to flaviviruses or togavirus. A majority of the cases of transfusion-related hepatitis that are non-A, non-B appear to be caused by hepatitis $C$. Many, but not all, donors capable of transmitting hepatitis $C$ will have evidence of elevated ALT, and before the emergence of a specific antibody test, an elevated ALT was used as a screening test in the hope of reducing the incidence of transfusionassociated hepatitis. The current test for hepatitis $C$ depends on an antibody to the virus that is not as precise as the antigenic markers of viral replication currently available in hepatitis $B$, and therefore our knowledge of this organism is less complete. ${ }^{6}$

The antibody test for hepatitis $C$ has become available only recently; therefore, patients who have required large numbers of blood transfusions are often found to be infected with hepatitis $C$ virus. Because these blood products have been efficiently screened for hepatitis $B$, it is not surprising that hepatitis $C$ virus infection accounts for most of the chronic liver disease observed in this population. There is also evidence of vertical transmission of hepatitis $C$ from mother to child, although the role of this perinatally acquired infection is unclear.

It appears that the hepatitis $C$ virus is much less infectious than the hepatitis $B$ virus. Relatively large quantities of infective material are necessary for infection. It has been estimated that half of the people infected with hepatitis $C$ will develop chronic hepatitis for at least a year and that $20 \%$ will eventually develop cirrhosis. Current therapy is primarily supportive, although interferon has had some possible successes in adults. In most epidemiologic studies to date, $40 \%$ of non- $A$, non-B hepatitis cases do not have an identifiable source.

\section{References}

1. Verhave M, Winter HS, Grand RJ: Azathioprine in the treatment of children with inflammatory bowel disease. J Pediatr 1990; 117:809-814.

2. Meyers $\mathrm{S}$, Sachar $\mathrm{DB}$, Present $\mathrm{DH}$, Janowitz $\mathrm{HD}$ : Olsalazine sodium in the treatment of ulcerative colitis among patients intolerant of sulfasalazine. Gastroenterology 1985; 88:717-722.

3. Jiranek GC, Kinmey MB, Saunders DR, Willson RA, Shanahan W, Silverstein FE: Misoprostol reduces gastroduodenal injury from one week of aspirin: An endoscopic study. Gastroenterology 1989; 96(pt 2, suppl 2):656-661.

4. Robinson P. Sly PD: Placebo-controlled trial of misoprostol in cystic fibrosis. J Pediatr Gastroenterol Nutr 1990; 11:37-40.

5. Kilbridge PM, Dahms BB, Czinn SJ: Campylobacter pylori-associated gastritis and peptic ulcer disease in children. Am J Dis Child 1988; 142:1149-1152

6. Czinn SJ, Bertram TA, Murray PD, Yang P: Relationship between gastric inflammatory response and symptoms in patients infected with Helicobacter pylori. Scand J Gastroenterol 1991: (suppl) 181:33-37. 